\title{
Effect of Different Planting Methods and Weed Management Practices on Yield and Economic of Mustard (Brassica juncea L.) and Weed Population, Weed Dry Matter Accumulation, Weed Control Efficiency, Weed Index, Nutrient Content and Uptake by Weeds
}

\author{
Rameti Jangir ${ }^{*}$, L.K. Arvadia and Deep Singh Rajpurohit
}

Department of Agronomy, N. M. College of Agriculture, Navsari Agricultural University, Navsari-396450, Gujarat, India

*Corresponding author

\section{Keywords}

Economics, Planting method, Weed control efficiency, Weed dry matter, Weed index, Weed management,

Weed population,

Yield of mustard crop

Article Info

Accepted:

24 January 2018

Available Online:

10 February 2018

\section{A B S T R A C T}

A field experiment was laid out on mustard (Brassica juncea L.) with different planting methods and weed management practices on College Farm of N. M. College of Agriculture, Navsari Agricultural University, Navsari throughout rabi season of 2014-15. Treatments were replicated thrice as per randomized block design with factorial concept. The treatment $45 \mathrm{~cm} \times 10 \mathrm{~cm}$ with normal planting $\left(\mathrm{P}_{2}\right)$ registered significantly higher values of seed yield $\left(1851 \mathrm{~kg} \mathrm{ha}^{-1}\right)$, stover yield $\left(3808 \mathrm{~kg} \mathrm{ha}^{-1}\right)$ with highest net realization of ₹ $52874 \mathrm{ha}^{-1}$ and benefit: cost ratio (BCR) of 3.29 remained at par with treatment of $30 / 60 \mathrm{~cm} \times 10 \mathrm{~cm}$ with paired row planting $\left(\mathrm{P}_{3}\right)$ in case of seed and stover yield with net return of ₹ $49614 \mathrm{ha}^{-1}$ with BCR of 3.15. Total weed population and dry weight of total weeds were significantly lowest under narrow row spacing of $30 \mathrm{~cm}$ x $10 \mathrm{~cm}$ with normal planting $\left(\mathrm{P}_{1}\right)$. Likewise, significantly higher values of seed yield $\left(2085 \mathrm{~kg} \mathrm{ha}^{-1}\right)$, stover yield $\left(4230 \mathrm{~kg} \mathrm{ha}^{-1}\right)$ with maximum economical gain of ₹ $60458 \mathrm{ha}^{-1}$ and BCR of 3.41 and lowest values of total weed population, dry weight of total weeds, weed index with better weed control efficiency were obtained with application of Pendimethalin@1.0 $\mathrm{kg} \mathrm{ha}^{-1}$ as PE + Quizalofop - P - ethyl @ $0.04 \mathrm{~kg} \mathrm{ha}^{-1}$ at $20 \mathrm{DAS}+\mathrm{HW}$ and IC at $40 \mathrm{DAS}\left(\mathrm{W}_{4}\right)$ but it was at par with treatments of $\mathrm{W}_{3}$ (Pendimethalin @ $1.0 \mathrm{~kg} \mathrm{ha}^{-1}$ as PE + HW and IC at 40 DAS) along with net realization by $₹ 59587 \mathrm{ha}^{-1}$ with BCR of 3.53 and $\mathrm{W}_{2}$ (Pendimethalin @ $1.0 \mathrm{~kg} \mathrm{ha}^{-1}$ as PE + Quizalofop - P - ethyl @ $0.04 \mathrm{~kg} \mathrm{ha}^{-1}$ at $20 \mathrm{DAS}$ ) along with net realization $56082 \mathrm{ha}^{-1}$ with BCR of 3.51 in case of seed and stover yield. The chemical analysis of weed plant indicated that the removal of nutrients through weeds was lowest in narrow row spacing of $30 \mathrm{~cm} \times 10 \mathrm{~cm}\left(\mathrm{P}_{1}\right)$ and treatment $\mathrm{W}_{4}$ viz., Pendimethalin @ $1.0 \mathrm{~kg}$ $\mathrm{ha}^{-1}$ as PE + Quizalofop - P - ethyl @ $0.04 \mathrm{~kg} \mathrm{ha}^{-1}$ at 20 DAS + HW and IC at 40 DAS. Hence the crop sown with wider row spacing and weed management with integration of all methods showed favourable growth leads to higher yield of mustard.

\section{Introduction}

Rapeseed/mustard is one of the most important oilseed crops of global economic importance and belongs to the family Cruciferae. It is the third major oilseed crop of India, ranking after groundnut and soybean, with around 23 per cent share of total oilseed 
production (Rajak et al., 2011). India's rank first in area $(20.2 \%)$ and second in production $(10.7 \%)$ after China in rapeseed and mustard growing countries of the world (Annon, 2014). Oilseed crops occupy an area of 28.2 million hectares with total production of 32.9 million tonnes and productivity $1149 \mathrm{~kg} \mathrm{ha}^{-1}$ at national level. Mustard alone occupies an area of 6.5 million hectare with the total production of 7.8 million tonnes and productivity of 1208 $\mathrm{kg} \mathrm{ha}{ }^{-1}$ during 2013 among the different oilseed crops (Annon, 2013).

The oil is utilised for human consumption throughout northern India in cooking and frying purposes. The whole seed is used as condiment in the preparation of pickles and for flavouring curries and vegetables.

The mustard oil is also used in preparing vegetable ghee, hair oil, medicines, soaps, lubricating oil and in tanning industries. The oil content in mustard seeds varies from 37-49 per cent (Bhowmik et al., 2014). The oil cake is left after extraction is utilized as cattle feed and manure. It contains $25-30$ per cent crude protein, 5 per cent nitrogen, 1.8-2.0 per cent phosphorous and 1.0-1.2 per cent potassium.

Growth, development and final yield are mainly affected by the space available to plants; however, the precise and exact response will be species and cultivar specific. So, it is imperative to adjust plant population through planting method which may help in avoiding excessive crowding and thereby enabling the plants to utilize these resources more effectively and efficiently resulting in increased production. Higher plant population per unit area beyond an optimum limit results in competition among the plants for natural resources, resulting weaker plant and may cause severe lodging (Kumar et al., 2004). While, low density population produce more branches that carry fertile pods, thus prolonging the seed development phase.
Weed competition in Indian mustard is more serious during early stage; because crop growth during winter (rabi) season remains slow during the first 4-6 weeks after sowing (Chauhan et al., 2005). Prevalence of higher temperature and availability of adequate moisture due to irrigation and adequate preparatory tillage provide most congenial conditions for quick growth of weeds in rabi season. Weeds are one of the major constraints for the poor yield of mustard crop as they compete with the crop plants for moisture, nutrients, light and space. Approximately, 2030 per-cent yield reduction cause by weeds in rape/mustard crop (Singh et al., 2010). Weed management is another important strategy for higher yield realization. This necessitates that a systematic study on weed dynamics in such crops is essential for strategic weedmanagement planning. Further, a weed-control method to be accepted by the farmers must be agronomically feasible, economically viable and should be under the farmer's manageable capacity. Among the various methods of weeds control, the traditional methods of weed control like hand-weeding, hoeing, and intercultural operations have many limitations. These are very often back breaking, costly, time-consuming and needs so much of human labour/resource. Besides, such weed-control measures are often also rendered uncertain due to interference of rains. The use of the herbicides is, thus, the only resort as it offers a good scope for timely and adequate control of weeds. The present study was therefore undertaken to assess the influence of planting methods and weed management practices on weeds and mustard crop.

\section{Materials and Methods}

A field experiment was conducted at College Farm of Navsari Agricultural University, Navsari throughout rabi season of 2014-15. Navsari Agricultural University campus is geographically located at $20^{\circ}-57^{\prime} \mathrm{N}$ latitude 
and $72^{0}-54^{\prime} \mathrm{E}$ longitude at an altitude of 10 meters above the mean sea level. According to agro-climatic condition, Navsari is located in south Gujarat heavy rainfall zone-I (Agroecological situation-III). The climate of this zone is typically tropical, characterized by humid and warm monsoon with heavy rain, quite cold winter and fairly hot summer. The average annual rainfall of the tract is about $1500 \mathrm{~mm}$. The soil of the experimental field was clayey in texture and showed low, medium and high rating for available nitrogen (197.26 kg ha $\left.{ }^{-1}\right)$, phosphorus (30.93 kg ha ${ }^{-1}$ ) and potassium $\left(369.80 \mathrm{~kg} \mathrm{ha}^{-1}\right)$, respectively. The soil was found slightly alkaline $(\mathrm{pH} 7.8)$ with normal electrical conductivity $(0.36$ $\left.\mathrm{dsm}^{-1}\right)$.

The experiment was conducted with total fifteen treatment combinations consisting of three levels of planting methods viz., $\mathrm{P}_{1}: 30$ $\mathrm{cm} \times 10 \mathrm{~cm}$ with normal planting, $\mathrm{P}_{2}: 45 \mathrm{~cm} \mathrm{x}$ $10 \mathrm{~cm}$ with normal planting and $\mathrm{P}_{3}: 30 / 60 \mathrm{~cm}$ $\mathrm{x} 10 \mathrm{~cm}$ with paired row planting and five levels of weed management practices viz., $\mathrm{W}_{0}$ : Weedy check, $\mathrm{W}_{1}$ : HW and IC at 20 and 40 DAS, $W_{2}$ : Pendimethalin @ $1.0 \mathrm{~kg} \mathrm{ha}^{-1}$ as PE + Quizalofop - P - ethyl @ $0.04 \mathrm{~kg} \mathrm{ha}^{-1}$ at 20 DAS, W : $_{3}$ Pendimethalin @ $1.0 \mathrm{~kg} \mathrm{ha}^{-1}$ as PE + HW and IC at 40 DAS and $\mathrm{W}_{4}$ : Pendimethalin @ $1.0 \mathrm{~kg} \mathrm{ha}^{-1}$ as PE + Quizalofop - P - ethyl @0.04 kg ha ${ }^{-1}$ at 20 DAS + HW and IC at 40 DAS, were evaluated with randomized block design with factorial concept and replicated thrice.

The investigation was carried out with "GDM4" variety of mustard and seeds were treated with Dithane M-45 fungicide @ $3 \mathrm{~g} \mathrm{~kg}^{-1}$. Spraying of herbicide pre-emergence Pendimethalin and post emergence Quizalofop -P-ethyl was done at 2 DAS and 20 DAS respectively as per treatment. The observations for different characters were recorded at different periodical intervals. The statistical analysis of data was carried out through the procedure appropriate to the randomized block design with factorial concept of the experiment as described by Panse and Sukhatme (1967).

The number of monocots, dicots and sedges were counted and recorded from the demarcated $1 \mathrm{~m}^{2}$ area of each net plot at 20 , 40 DAS and at harvest and expressed the weed population as plant $\mathrm{m}^{2}$. Data on weed population showed considerable variation and hence the data were subjected to square root $(\sqrt{X+0.5})$ transformation before statistical analysis (Gomez and Gomez, 1984).

In case of weed dry matter accumulation the weed samples were collected from $1.0 \mathrm{~m}^{2}$ area and expressed as $\mathrm{g} \mathrm{m}^{-2}$ and finally at the time of harvest from entire net plot area of each plot and expressed as $\mathrm{kg} \mathrm{ha}^{-1}$.

After removing the roots, the above ground plant parts and whole weed samples were first sun dried and finally oven dried at $65{ }^{0} \mathrm{C}$ for 72 hours up to constant dry weight.

Representative samples of weeds were taken separately from each plot for estimation of $\mathrm{N}$, $\mathrm{P}$ and $\mathrm{K}$ content. The samples were oven dried at $60^{\circ} \mathrm{C}$ for $24 \mathrm{hrs}$, powdered by mechanical grinder and analyzed for respective nutrient content using following procedures.

The uptake of nitrogen $(\mathrm{N})$, phosphorus (P) and potassium $(\mathrm{K})$ by weeds were worked out by using the following formula:

Nutrient uptake by Weed plant $\left(\mathrm{kg} \mathrm{ha}^{-1}\right)=$

$\%$ Nutrient content $\mathrm{x}$ Weed biomass At harvest $\left(\mathrm{kg} \mathrm{ha}^{-1}\right)$

100

The weed control efficiency was calculated by using the following formula (Kondap and Upadhyay, 1985). 


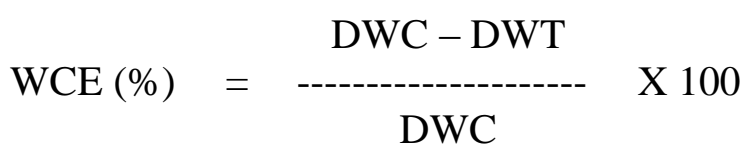

Where, DWC and DWT were the weed dry weight in control and treated plots, respectively.

Weed index (\%) was estimated by using the formula suggested by Kumar and Gill (1969) and expressed as:

$\mathrm{WI}(\%)=\frac{\mathrm{X}-\mathrm{Y}}{\mathrm{X}}$

Where, WI = Weed index $(\%)$

$\mathrm{X}=$ Maximum yield from the treatment or weed free plot

$\mathrm{Y}=$ Yield from the treatment for which weed competition index is to be estimated or treated plot

The crop was harvested manually when more than $80 \%$ of siliquae changed their colour from green to light yellow. Economics of different treatments was worked out on the basis of input and output at the prevailing market rates. The net realization was worked out by deducting the total cost of cultivation from gross realization per hectare for each treatment and recorded accordingly. The benefit cost ratio (BCR) was calculated as follows:

$$
\mathrm{BCR}=\frac{\text { Gross realization }}{\text { Total cost of cultivation }}
$$

\section{Results and Discussion}

\section{Weed flora}

During crop season the experimental field was mainly infested with monocot weeds viz.,
Echinochloa crusgalli (L.), Digitaria sanguinalis (L.) Scop, Eragrostis barbinodis, and Cynodon dactylon (L.) Pers. Similarly, dicot weeds viz., Convolvulus arvensis (L.), Digera arvensis (Forsk.), Achyranthes argentea, Achyranthes sessilis, Corchorus triflorus, Abutilon indicum and Boerhavia repens and the prominent weed amongst sedges was found to be Cyperus rotundus (L.).

\section{Effect of planting methods}

The treatment $\mathrm{P}_{2}(45 \mathrm{~cm} \times 10 \mathrm{~cm}$ with normal planting) registered maximum seed yield (1851 kg ha $\left.{ }^{-1}\right)$ and stover yield (3808 $\mathrm{kg} \mathrm{ha}^{-1}$ ) remained at par with treatment $\mathrm{P}_{3}(30 / 60 \mathrm{~cm} \mathrm{x}$ $10 \mathrm{~cm}$ with paired row planting). Both these treatments were found significantly superior to treatment $\mathrm{P}_{1} 30 \mathrm{~cm} \times 10 \mathrm{~cm}$ with normal planting. The magnitude of increase in seed, stover yield in mustard 15.2, 19.3\% and 10.3, $13.1 \%$, under $\mathrm{P}_{2}$ and $\mathrm{P}_{3}$, respectively over closer spacing with normal planting treatment $\mathrm{P}_{1}$. It was probably due to optimum plant population per unit area which gave optimum yield per plant and lower plant competition. The wider row spacing improved individual plant yield and yield per unit area is the resultant of cumulative yield from individual plants per unit area. A narrow row spacing sown crops have more number of plants per unit area and reduction in yield per plant might not to be compensated with yield from more number of plants per unit area. These results are in agreement with those of Pyare et al., (2008) at Kanpur; Muhammad et al., (2012). This result was also supported by Rajput (2012) and recorded higher seed and stover yield under $30 / 60 \mathrm{~cm}$ x $10 \mathrm{~cm}$ with paired row planting which was at par to $45 \mathrm{~cm}$ x $10 \mathrm{~cm}$ row spacing at Firozabad (UP).

The effect on weed population i.e. monocots, dicots and sedges due to planting methods at 20 DAS was found to be non-significant. While, significantly least number of total 
weed $\left(30.60 \mathrm{~m}^{-2}\right)$ was found with narrow spacing i.e. $30 \mathrm{~cm} \mathrm{x} 10 \mathrm{~cm}$ with normal planting (P1). At 40 DAS least number of monocots, dicots, sedges and total weed population were registered under the treatment $\mathrm{P}_{1}$ but it was at par with treatment $\mathrm{P}_{2}(45 \mathrm{~cm} \mathrm{x}$ $10 \mathrm{~cm}$ with normal planting) only in case of dicots and sedges. Whereas, at harvest minimum number of monocots, dicots, sedges and total weed population were also recorded under the treatment of $\mathrm{P}_{1}$ but it was at par with treatment $\mathrm{P}_{2}$ in case of monocots, dicots and sedges.

That might be due to more number of plants per unit area in narrow row spacing lead to shading effect resulting from the crop canopy, limits availability of resources required for weed germination, emergence and growth.

This effect is more pronounced in $30 \mathrm{~cm} \mathrm{x} 10$ $\mathrm{cm}$ row spacing $\left(\mathrm{P}_{1}\right)$. Weed biomass reduction at this period can be explained by the reduction in total incoming photosynthetic active radiation (PAR) reaching the ground and indicates that narrow inter-row spacing increased the competitiveness of the mustard with weeds. These results are in line with those reported by Patel (2000) in sugarcane, Hussein et al., (2008) in maize; Takim and Adereti (2012) in soybean and Bakhat and Khan (2014) in tomato.

At 20, 40 DAS and at harvest significantly lower dry weight $\left(9.53,20.99 \mathrm{~g} \mathrm{~m}^{-2}\right.$ and $269.59 \mathrm{~kg} \mathrm{ha}^{-1}$, respectively) of total weeds were observed under treatment $P_{1}$ but it was statistically at par with treatment $\mathrm{P}_{2}(45 \mathrm{~cm} \mathrm{x}$ $10 \mathrm{~cm}$ with normal planting) only at 20 DAS.

A perusal of data presented in Table 4 indicated that the $\mathrm{N}, \mathrm{P}$ and $\mathrm{K}$ content (per cent) in weeds were recorded to be nonsignificant. The results indicated that narrow spacing of $30 \mathrm{~cm} \times 10 \mathrm{~cm}$ with normal planting $\left(\mathrm{P}_{1}\right)$ was found conductive for lower nutrient uptake $\left(2.40 \mathrm{~kg} \mathrm{ha}^{-1} \mathrm{~N}, 0.88 \mathrm{~kg} \mathrm{ha}^{-1} \mathrm{P}\right.$ and $2.80 \mathrm{~kg} \mathrm{ha}^{-1} \mathrm{~K}$ ) by weeds of mustard crop. While, maximum nutrient uptake was found with wider row spacing of $30 / 60 \mathrm{~cm} \times 10 \mathrm{~cm}$ $\left(\mathrm{P}_{3}\right)$ with paired row planting. That might be due to higher weed biomass produced in $\mathrm{P}_{3}$ due to comparatively better availability of sufficient moisture, nutrients, space and light ultimately resulted into significantly higher $\mathrm{N}$, $\mathrm{P} \& \mathrm{~K}$ uptake with these treatments. Almost similar result was observed by Patel (2000) in sugarcane crop.

Highest net return of ₹ $52874 \mathrm{ha}^{-1}$ and benefit: cost ratio (BCR) of 3.29 was secured with treatment of $\mathrm{P}_{2}(45 \mathrm{~cm} \mathrm{x} 10 \mathrm{~cm}$ with normal planting) followed by $\mathrm{P}_{3}(30 / 60 \mathrm{~cm} \mathrm{x}$ $10 \mathrm{~cm}$ with paired row planting) with net return of ₹ 49614 ha $^{-1}$ with BCR of 3.15.

This was attributed to remarkable increase in gross realization with comparatively lower cost in this treatment resulted in higher net realization and BCR. These results are conformity with those reported by Singh et al., (2006); Pyare et al., (2008) and Rajput (2012). While, Iraddi (2008) reported contradictory results in this regard and concluded that narrow row spacing $30 \mathrm{~cm}$ gave higher net returns in mustard crop.

\section{Effect of weed management}

Treatment $\mathrm{W}_{4}$ i.e. Pendimethalin@ $1.0 \mathrm{~kg}$ ha ${ }^{1}$ as PE + Quizalofop - P - ethyl @ $0.04 \mathrm{~kg} \mathrm{ha}^{-1}$ at $20 \mathrm{DAS}+\mathrm{HW}$ and IC at 40 DAS recorded significantly higher seed yield (2085 kg ha ${ }^{-1}$ ) and stover yield $\left(4230 \mathrm{~kg} \mathrm{ha}^{-1}\right)$ but it was at par with treatment $\mathrm{W}_{3}$ (Pendimethalin@1.0 $\mathrm{kg} \mathrm{ha}^{-1}$ as PE + HW and IC at 40 DAS) and $\mathrm{W}_{2}$ (Pendimethalin@ $1.0 \mathrm{~kg} \mathrm{ha}^{-1}$ as $\mathrm{PE}+$ Quizalofop - P - ethyl @ $0.04 \mathrm{~kg} \mathrm{ha}^{-1}$ at 20 DAS). The magnitude of increase in seed yield $\mathrm{kg} \mathrm{ha}^{-1}$ under treatments $\mathrm{W}_{4}, \mathrm{~W}_{3}$ and $\mathrm{W}_{2}$ were 109.3, 103.5 and 92.1 per cent, respectively over weedy check treatment $\left(\mathrm{W}_{0}\right)$. 
Table.1 Seed yield $\left(\mathrm{kg} \mathrm{ha}^{-1}\right)$, stover yield $\left(\mathrm{kg} \mathrm{ha}^{-1}\right)$ and economics of mustard as influenced by different planting methods and weed management treatments

\begin{tabular}{|c|c|c|c|c|c|}
\hline Treatments & $\begin{array}{l}\text { Seed yield } \\
\left(\mathrm{kg} \mathrm{ha}^{-1}\right)\end{array}$ & $\begin{array}{c}\text { Stover yield } \\
\left(\mathrm{kg} \mathrm{ha}^{-1}\right)\end{array}$ & $\begin{array}{c}\text { Gross realization } \\
\left(\mathrm{Rs} \mathrm{ha}^{-1}\right)\end{array}$ & $\begin{array}{l}\text { Net realization } \\
\left(\mathbf{R s ~ h a}^{-1}\right)\end{array}$ & BCR \\
\hline \multicolumn{6}{|c|}{ Planting methods (P) } \\
\hline$\overline{P_{1}}$ & 1607 & 3191 & 65876 & 42136 & 2.77 \\
\hline $\mathbf{P}_{2}$ & 1851 & 3808 & 75944 & 52874 & 3.29 \\
\hline $\mathbf{P}_{3}$ & 1772 & 3608 & 72684 & 49614 & 3.15 \\
\hline S.Em. \pm & 52.05 & 113.23 & - & - & - \\
\hline C.D. at 5\% & 150.76 & 327.96 & - & - & - \\
\hline \multicolumn{6}{|c|}{ Weed management practices (W) } \\
\hline $\mathrm{W}_{0}$ & 996 & 2181 & 40931 & 21971 & 2.16 \\
\hline $\mathrm{W}_{1}$ & 1697 & 3366 & 69563 & 45203 & 2.86 \\
\hline $\mathrm{W}_{2}$ & 1913 & 3838 & 78439 & 56082 & 3.51 \\
\hline $\mathbf{W}_{3}$ & 2027 & 4063 & 83112 & 59587 & 3.53 \\
\hline $\mathrm{W}_{4}$ & 2085 & 4230 & 85515 & 60458 & 3.41 \\
\hline S.Em. \pm & 67.20 & 146.18 & - & - & - \\
\hline C.D. at $5 \%$ & 194.63 & 423.39 & - & - & - \\
\hline C.V. $\%$ & 11.56 & 12.40 & - & - & - \\
\hline Interaction & NS & NS & - & - & - \\
\hline
\end{tabular}

Table.2 Weed population at 20,40 DAS and at harvest as influenced by different planting methods and weed management treatments

\begin{tabular}{|c|c|c|c|c|c|c|c|c|c|c|c|c|}
\hline \multirow{2}{*}{$\begin{array}{l}\text { Treatment } \\
\text { s }\end{array}$} & \multicolumn{4}{|c|}{ At 20 DAS } & \multicolumn{4}{|c|}{ At 40 DAS } & \multicolumn{4}{|c|}{ At harvest } \\
\hline & Monocot & Dicot & $\begin{array}{c}\text { Sedg } \\
\text { e }\end{array}$ & Total & $\begin{array}{c}\text { Monoco } \\
t\end{array}$ & Dicot & Sedge & Total & $\begin{array}{c}\text { Monoco } \\
t\end{array}$ & Dicot & Sedge & Total \\
\hline \multicolumn{13}{|c|}{ Planting methods (P) } \\
\hline$\overline{\mathbf{P}_{1}}$ & $3.26(9.87)$ & $\begin{array}{c}2.70 \\
(7.20)\end{array}$ & $\begin{array}{c}3.67 \\
(13.53)\end{array}$ & $\begin{array}{c}5.52 \\
(30.60)\end{array}$ & $\begin{array}{c}3.83 \\
(14.93)\end{array}$ & $\begin{array}{c}3.61 \\
(12.80)\end{array}$ & $\begin{array}{c}4.47 \\
(19.80)\end{array}$ & $\begin{array}{c}6.85 \\
(47.53)\end{array}$ & $\begin{array}{c}5.29 \\
(28.00)\end{array}$ & $\begin{array}{c}3.92 \\
(15.07)\end{array}$ & $\begin{array}{c}5.64 \\
(31.87)\end{array}$ & $\begin{array}{c}8.62 \\
(74.93)\end{array}$ \\
\hline$\overline{\mathbf{P}_{2}}$ & $\begin{array}{c}3.45 \\
(11.13)\end{array}$ & $\begin{array}{c}2.82 \\
(7.80)\end{array}$ & $\begin{array}{c}3.83 \\
(14.93)\end{array}$ & $\begin{array}{c}5.80 \\
(33.87)\end{array}$ & $\begin{array}{c}4.32 \\
(19.27)\end{array}$ & $\begin{array}{c}3.79 \\
(14.13)\end{array}$ & $\begin{array}{c}4.71 \\
(22.13)\end{array}$ & $\begin{array}{c}7.38 \\
(55.53)\end{array}$ & $\begin{array}{c}5.50 \\
(30.33)\end{array}$ & $\begin{array}{c}4.13 \\
(16.93)\end{array}$ & $\begin{array}{c}5.88 \\
(34.80)\end{array}$ & $\begin{array}{c}9.01 \\
(82.07)\end{array}$ \\
\hline$\overline{\mathbf{P}_{3}}$ & $\begin{array}{c}3.54 \\
(11.27)\end{array}$ & $\begin{array}{c}2.88 \\
(8.13)\end{array}$ & $\begin{array}{c}3.88 \\
(15.33)\end{array}$ & $\begin{array}{c}5.93 \\
(34.73)\end{array}$ & $\begin{array}{c}4.84 \\
(25.00)\end{array}$ & $\begin{array}{c}3.93 \\
(15.20)\end{array}$ & $\begin{array}{c}4.83 \\
(23.40)\end{array}$ & $\begin{array}{c}7.85 \\
(63.60)\end{array}$ & $\begin{array}{c}5.67 \\
(32.13)\end{array}$ & $\begin{array}{c}4.31 \\
(18.47)\end{array}$ & $\begin{array}{c}6.10 \\
(37.40)\end{array}$ & $\begin{array}{c}9.33 \\
(88.00)\end{array}$ \\
\hline S.Em. \pm & 0.09 & 0.05 & 0.06 & 0.07 & 0.08 & 0.06 & 0.09 & 0.07 & 0.09 & 0.07 & 0.10 & 0.08 \\
\hline C.D. at 5\% & NS & NS & NS & 0.20 & 0.23 & 0.19 & 0.27 & 0.19 & 0.27 & 0.21 & 0.28 & 0.22 \\
\hline \multicolumn{13}{|c|}{ Weed management practices (W) } \\
\hline$\overline{\mathrm{W}_{0}}$ & $\begin{array}{c}4.19 \\
(16.89)\end{array}$ & $\begin{array}{c}3.39 \\
(11.00)\end{array}$ & $\begin{array}{c}4.71 \\
(21.78)\end{array}$ & $\begin{array}{c}7.09 \\
(49.67)\end{array}$ & $\begin{array}{c}5.95 \\
(35.56)\end{array}$ & $\begin{array}{c}4.49 \\
(19.78)\end{array}$ & $\begin{array}{c}5.68 \\
(32.11)\end{array}$ & $\begin{array}{c}9.35 \\
(87.44)\end{array}$ & $\begin{array}{c}6.70 \\
(44.44)\end{array}$ & $\begin{array}{c}5.03 \\
(25.00)\end{array}$ & $\begin{array}{c}7.33 \\
(53.44)\end{array}$ & $\begin{array}{c}11.10 \\
(122.89)\end{array}$ \\
\hline$\overline{W_{1}}$ & $\begin{array}{c}4.36 \\
(18.00)\end{array}$ & $\begin{array}{c}3.63 \\
(12.67)\end{array}$ & $\begin{array}{c}4.87 \\
(23.33)\end{array}$ & $\begin{array}{c}7.42 \\
(54.00)\end{array}$ & $\begin{array}{c}5.05 \\
(25.56)\end{array}$ & $\begin{array}{c}4.11 \\
(16.44)\end{array}$ & $\begin{array}{c}4.87 \\
(23.33)\end{array}$ & $\begin{array}{c}8.09 \\
(65.33)\end{array}$ & $\begin{array}{c}5.68 \\
(31.89)\end{array}$ & $\begin{array}{c}4.16 \\
(16.89)\end{array}$ & $\begin{array}{c}5.88 \\
(34.22)\end{array}$ & $\begin{array}{c}9.13 \\
(83.00)\end{array}$ \\
\hline$\overline{\mathrm{W}_{2}}$ & $2.85(6.56)$ & $\begin{array}{c}2.48 \\
(5.67)\end{array}$ & $\begin{array}{c}3.17 \\
(9.56)\end{array}$ & $\begin{array}{c}4.83 \\
(21.78)\end{array}$ & $\begin{array}{c}3.25 \\
(10.11)\end{array}$ & $\begin{array}{c}3.29 \\
(10.33)\end{array}$ & $\begin{array}{c}4.06 \\
(16.00)\end{array}$ & $\begin{array}{c}6.07 \\
(36.44)\end{array}$ & $\begin{array}{c}5.24 \\
(27.00)\end{array}$ & $\begin{array}{c}3.88 \\
(14.67)\end{array}$ & $\begin{array}{c}5.51 \\
(30.00)\end{array}$ & $\begin{array}{c}8.49 \\
(71.67)\end{array}$ \\
\hline$\overline{W_{3}}$ & $3.38(7.44)$ & $\begin{array}{c}2.11 \\
(4.00)\end{array}$ & $\begin{array}{c}3.02 \\
(8.67)\end{array}$ & $\begin{array}{c}4.91 \\
(20.11)\end{array}$ & $\begin{array}{c}4.34 \\
(18.56)\end{array}$ & $\begin{array}{c}3.76 \\
(13.78)\end{array}$ & $\begin{array}{c}4.62 \\
(20.89)\end{array}$ & $\begin{array}{c}7.31 \\
(53.22)\end{array}$ & $\begin{array}{c}5.01 \\
(24.67)\end{array}$ & $\begin{array}{c}3.83 \\
(14.22)\end{array}$ & $\begin{array}{c}5.38 \\
(28.56)\end{array}$ & $\begin{array}{c}8.23 \\
(67.44)\end{array}$ \\
\hline$\overline{W_{4}}$ & $2.30(4.89)$ & $\begin{array}{c}2.39 \\
(5.22)\end{array}$ & $\begin{array}{c}3.19 \\
(9.67)\end{array}$ & $\begin{array}{c}4.50 \\
(19.78)\end{array}$ & $\begin{array}{c}3.05 \\
(8.89)\end{array}$ & $\begin{array}{c}3.22 \\
(9.89)\end{array}$ & $\begin{array}{c}4.12 \\
(16.56)\end{array}$ & $\begin{array}{c}5.98 \\
(35.33)\end{array}$ & $\begin{array}{c}4.81 \\
(22.78)\end{array}$ & $\begin{array}{c}3.71 \\
(13.33)\end{array}$ & $\begin{array}{c}5.26 \\
(27.22)\end{array}$ & $\begin{array}{c}7.98 \\
(63.33)\end{array}$ \\
\hline S.Em. \pm & 0.11 & 0.07 & 0.08 & 0.09 & 0.10 & 0.08 & 0.12 & 0.08 & 0.12 & 0.10 & 0.12 & 0.10 \\
\hline C.D. at 5\% & 0.32 & 0.19 & 0.23 & 0.26 & 0.29 & 0.24 & 0.35 & 0.24 & 0.35 & 0.28 & 0.36 & 0.28 \\
\hline C.V. \% & 9.75 & 6.97 & 6.37 & 4.61 & 6.97 & 6.64 & 7.65 & 3.43 & 6.56 & 6.95 & 6.27 & 3.25 \\
\hline Interaction & NS & NS & NS & NS & $\mathrm{P} \times \mathrm{W}$ & NS & NS & $\mathrm{P} \times \mathrm{W}$ & NS & NS & NS & NS \\
\hline
\end{tabular}

Data in parentheses indicate actual value and those outside are $\sqrt{\mathrm{x}+0.5}$ transformed value. 
Table.3 At 40 DAS mean number of monocots and total weeds in mustard crop as influenced by interaction effect of different planting methods and weed management treatments

\begin{tabular}{|c|c|c|c|c|c|}
\hline \multirow{2}{*}{$\begin{array}{l}\text { Planting } \\
\text { methods (P) }\end{array}$} & \multicolumn{5}{|c|}{ Weed management (W) } \\
\hline & $\mathbf{W}_{0}$ & $\mathbf{W}_{1}$ & $\mathbf{W}_{2}$ & $\mathbf{W}_{3}$ & $\mathbf{W}_{4}$ \\
\hline \multicolumn{6}{|c|}{ Numbers of monocot weeds at 40 DAS } \\
\hline $\mathbf{P}_{1}$ & 5.05 & 4.36 & 3.03 & 3.89 & 2.85 \\
\hline $\mathbf{P}_{2}$ & 5.89 & 4.97 & 3.29 & 4.30 & 3.13 \\
\hline$\overline{\mathbf{P}_{3}}$ & 6.91 & 5.82 & 3.44 & 4.84 & 3.19 \\
\hline \multicolumn{6}{|c|}{ S.Em. \pm 0.17 C.D. at $5 \% 0.50$} \\
\hline \multicolumn{6}{|c|}{ Numbers of total weeds at 40 DAS } \\
\hline$\overline{\mathbf{P}_{1}}$ & 8.42 & 7.54 & 5.84 & 6.77 & 5.70 \\
\hline $\mathbf{P}_{2}$ & 9.35 & 8.06 & 6.09 & 7.34 & 6.04 \\
\hline$\overline{\mathbf{P}_{3}}$ & 10.27 & 8.68 & 6.28 & 7.84 & 6.20 \\
\hline
\end{tabular}

Table.4 Dry weight of weeds, Weed control efficiency (WCE) and weed index (WI) as influenced by different planting methods and weed management treatments

\begin{tabular}{|c|c|c|c|c|c|c|c|}
\hline \multirow[t]{2}{*}{ Treatments } & \multicolumn{3}{|c|}{ Weed dry weight } & \multicolumn{3}{|c|}{ Weed control efficiency (\%) } & \multirow{2}{*}{$\begin{array}{l}\text { Weed index } \\
(\%)\end{array}$} \\
\hline & $\begin{array}{c}20 \text { DAS } \\
\left(\mathrm{g} \mathrm{m}^{-2}\right)\end{array}$ & $\begin{array}{l}40 \text { DAS } \\
\left(\mathrm{g} \mathrm{m}^{-2}\right)\end{array}$ & $\begin{array}{l}\text { At harvest } \\
\left(\mathrm{kg} \mathrm{ha}^{-1}\right)\end{array}$ & At 20 DAS & At 40 DAS & At harvest & \\
\hline \multicolumn{8}{|c|}{ Planting methods (P) } \\
\hline$\overline{\mathbf{P}_{1}}$ & 9.53 & 20.99 & 269.59 & - & - & - & - \\
\hline$\overline{\mathbf{P}_{2}}$ & 10.29 & 26.05 & 314.26 & - & - & - & - \\
\hline $\mathbf{P}_{3}$ & 11.16 & 28.41 & 352.17 & - & - & - & - \\
\hline S.Em. \pm & 0.28 & 0.81 & 10.77 & - & - & - & - \\
\hline C.D. at $5 \%$ & 0.81 & 2.35 & 31.21 & - & - & - & - \\
\hline \multicolumn{8}{|c|}{ Weed management practices (W) } \\
\hline$\overline{\mathbf{W}_{0}}$ & 17.19 & 49.93 & 682.26 & 0.00 & 0.00 & 0.00 & 52.21 \\
\hline$\overline{\mathbf{W}_{1}}$ & 10.47 & 24.64 & 290.89 & 39.11 & 50.66 & 57.36 & 18.62 \\
\hline $\mathbf{W}_{2}$ & 9.06 & 17.03 & 198.76 & 47.31 & 65.89 & 70.87 & 8.24 \\
\hline $\mathrm{W}_{3}$ & 8.31 & 19.61 & 219.81 & 51.66 & 60.74 & 67.78 & 2.79 \\
\hline$\overline{W_{4}}$ & 6.61 & 14.54 & 168.31 & 61.55 & 70.87 & 75.33 & 0.00 \\
\hline S.Em. \pm & 0.36 & 1.05 & 13.91 & - & - & - & - \\
\hline C.D. at 5\% & 1.05 & 3.03 & 40.29 & - & - & - & - \\
\hline C.V. \% & 10.52 & 12.49 & 13.37 & - & - & - & - \\
\hline Interaction & NS & NS & NS & - & - & - & - \\
\hline
\end{tabular}


Table.5 Nitrogen, phosphorus and potassium content (\%) and uptake by weeds $\left(\mathrm{kg} \mathrm{ha}^{-1}\right)$ as influenced by different planting methods and weed management treatments

\begin{tabular}{|c|c|c|c|c|c|c|}
\hline \multirow[t]{2}{*}{ Treatments } & \multicolumn{3}{|c|}{$\mathbf{N}, \mathbf{P} \& \mathrm{~K}$ content in weed (\%) } & \multicolumn{3}{|c|}{ N, P \& K uptake by weeds $\left(\mathrm{kg} \mathrm{ha}^{-1}\right)$} \\
\hline & $\mathbf{N}$ & $\mathbf{P}$ & $\mathbf{K}$ & $\mathbf{N}$ & $\mathbf{P}$ & $\mathbf{K}$ \\
\hline \multicolumn{7}{|c|}{ Planting methods (P) } \\
\hline $\mathbf{P}_{1}$ & 0.875 & 0.321 & 1.025 & 2.40 & 0.88 & 2.80 \\
\hline $\mathbf{P}_{2}$ & 0.894 & 0.333 & 1.032 & 2.86 & 1.07 & 3.27 \\
\hline $\mathbf{P}_{3}$ & 0.910 & 0.340 & 1.044 & 3.24 & 1.22 & 3.72 \\
\hline S.Em. \pm & 0.014 & 0.007 & 0.017 & 0.15 & 0.04 & 0.14 \\
\hline C.D. at $5 \%$ & NS & NS & NS & 0.43 & 0.112 & 0.40 \\
\hline \multicolumn{7}{|c|}{ Weed management practices (W) } \\
\hline $\mathrm{W}_{0}$ & 0.926 & 0.349 & 1.069 & 6.35 & 2.38 & 7.30 \\
\hline $\mathrm{W}_{1}$ & 0.917 & 0.339 & 1.058 & 2.67 & 1.00 & 3.09 \\
\hline $\mathrm{W}_{2}$ & 0.895 & 0.326 & 1.042 & 1.784 & 0.65 & 2.06 \\
\hline $\mathbf{W}_{3}$ & 0.872 & 0.326 & 1.021 & 1.92 & 0.72 & 2.24 \\
\hline $\mathrm{W}_{4}$ & 0.854 & 0.315 & 0.978 & 1.43 & 0.53 & 1.65 \\
\hline S.Em. \pm & 0.019 & 0.009 & 0.022 & 0.19 & 0.05 & 0.18 \\
\hline C.D. at $5 \%$ & NS & NS & NS & 0.55 & 0.15 & 0.51 \\
\hline C.V. \% & 6.270 & 7.700 & 6.360 & 20.24 & 4.73 & 16.21 \\
\hline Interaction & NS & NS & NS & NS & NS & NS \\
\hline
\end{tabular}

$\mathrm{P}_{1}: 30 \mathrm{~cm}$ x $10 \mathrm{~cm}$ with normal planting; $\mathrm{P}_{2}: 45 \mathrm{~cm}$ x $10 \mathrm{~cm}$ with normal planting; $\mathrm{P}_{3}: 30 / 60 \mathrm{~cm} \mathrm{x} 10 \mathrm{~cm}$ with paired row planting; $\mathrm{W}_{0}$ : Weedy check; $\mathrm{W}_{1}$ : HW and IC at 20 and $40 \mathrm{DAS} ; \mathrm{W}_{2}$ : Pendimethalin @ $1.0 \mathrm{~kg} \mathrm{ha}^{-1}$ as PE + Quizalofop - P - ethyl @ $0.04 \mathrm{~kg} \mathrm{ha}^{-1}$ at 20 DAS; $\mathrm{W}_{3}$ : Pendimethalin @ $1.0 \mathrm{~kg} \mathrm{ha}^{-1}$ as PE + HW and IC at 40 DAS and $\mathrm{W}_{4}$ : Pendimethalin@1.0 kg ha ${ }^{-1}$ as PE + Quizalofop - P - ethyl @ $0.04 \mathrm{~kg} \mathrm{ha}^{-1}$ at 20 DAS + HW and IC at 40 DAS.

\section{Representative samples of weeds}

\begin{tabular}{|l|l|l|l|}
\hline Sr. No. & Particular & Procedure used & Reference \\
\hline 1. & Nitrogen (\%) & Modified Kjeldahl's Method phoshoric acid & Jackson (1967) \\
\hline 2. & Phosphorus (\%) & $\begin{array}{l}\text { Vanadomolybdo phon } \\
\text { yellow colour method }\end{array}$ \\
\hline 3. & Potash (\%) & Flame photometric method & Jackson (1967) \\
\hline
\end{tabular}

The remarkable increase in seed and stover yield might be due to effective control of weeds, lower dry weight of weeds and higher weed control efficiency as well as lower weed index which cumulatively facilitated the crop to utilize more nutrients and water for better growth and development in terms of various growth attributing characters and yield attributing characters. These findings are in close agreement with those reported by Sarkar et al., (2005); Rathi et al., (2007); Kumar et al., (2012) as well as Adhikary and Ghosh (2014).
At 20 DAS, the lowest number of monocots $\left(4.89 \mathrm{~m}^{-2}\right)$ and total weeds $\left(19.78 \mathrm{~m}^{-2}\right)$ were recorded with the application of Pendimethalin @ $1.0 \mathrm{~kg} \mathrm{ha}^{-1}$ as PE + Quizalofop - P - ethyl @ $0.04 \mathrm{~kg} \mathrm{ha}^{-1}$ at 20 $\mathrm{DAS}+\mathrm{HW}$ and IC at $40 \mathrm{DAS}\left(\mathrm{W}_{4}\right)$. While, significantly lowest number of dicots (4.00 $\left.\mathrm{m}^{-2}\right)$ and sedges $\left(8.67 \mathrm{~m}^{-2}\right)$ were recorded with Pendimethalin@1.0 kg ha ${ }^{-1}$ as PE + HW and $\mathrm{IC}$ at $40 \mathrm{DAS}\left(\mathrm{W}_{3}\right)$ but it remained at par with $\mathrm{W}_{2}$ (Pendimethalin @ $1.0 \mathrm{~kg} \mathrm{ha}^{-1}$ as $\mathrm{PE}+$ Quizalofop - P - ethyl @ $0.04 \mathrm{~kg} \mathrm{ha}^{-1}$ at 20 DAS) and $\mathrm{W}_{4}$ (Pendimethalin @ $1.0 \mathrm{~kg} \mathrm{ha}^{-1}$ 
as PE + Quizalofop - P - ethyl @ $0.04 \mathrm{~kg} \mathrm{ha}^{-1}$ at $20 \mathrm{DAS}+\mathrm{HW}$ and IC at $40 \mathrm{DAS})(9.56$ and $9.67 \mathrm{~m}^{-2}$, respectively) in case of sedges only.

At 40 DAS, significantly minimum number of monocots, dicots and total weeds were recorded with treatment $\mathrm{W}_{4}$ while, lowest number of sedges were found with treatment $\mathrm{W}_{2}$. At harvest, Significantly minimum number of monocots, dicots, sedges and total weeds were observed in treatment $\mathrm{W}_{4}$ whereas, it was at par with treatment $\mathrm{W}_{2}$ and $\mathrm{W}_{3}$ in case of dicots and sedges but it was at par with the treatment $\mathrm{W}_{3}$ only with respect to monocots and total weeds (Table 2).

At earlier stage of crop growth, the lowest population of individual as well as total weeds was recorded randomly with pendimethalin treatments i.e. $\mathrm{W}_{4}, \mathrm{~W}_{3}$ and $\mathrm{W}_{2}$. This might be due to pendimethalin@1.0 kg ha ${ }^{-1}$ alone was found most effective to control the weeds in mustard at early crop growth stage. But at later stages of crop growth, the lowest population of individual as well as total weeds was recorded under treatment $\mathrm{W}_{4}$, which was closely followed by $\mathrm{W}_{3}$ and $\mathrm{W}_{2}$. As regards to herbicides, pendimethalin@1.0 kg ha ${ }^{-1}$ was found effective either followed by Quizalofop - P - ethyl@0.04 kg ha ${ }^{-1}$ at 20 DAS or HW and IC at 40 DAS or followed by both as compared to weedy check $\left(\mathrm{W}_{0}\right)$. Quizalofop $P$ - ethyl treatment remarkably reduced the population of monocots and sedges, while, population of dicots were reduced with pendimethalin application. The findings are confirmation with those reported by Banga et al., (2004) in Indian mustard; Yadav (2004) in Indian mustard; Rathi et al., (2007) in chickpea + mustard intercropping system and Kour et al., (2014) in chickpea + Indian mustard intercropping system.

The dry weight of weeds recorded at 20, 40 DAS and at harvest and noticed that treatment
$\mathrm{W}_{4}$ recorded the lower dry weight of weeds but it was found at par with treatment $\mathrm{W}_{2}$ at 40 DAS and at harvest except 20 DAS. Treatment $\mathrm{W}_{4}$ gave significantly lower dry weight of weeds $\left(6.61,14.54 \mathrm{~g} \mathrm{~m}^{-2}\right.$ and $168.31 \mathrm{~kg} \mathrm{ha}^{-1}$, respectively). Similar results were also reported by Banga et al., (2004) in Indian mustard; Yadav (2004) in Indian mustard; Sarkar et al., (2005) in mustard; Rathi et al., (2007) in chickpea + mustard intercropping system and Kour et al., (2014) in chickpea + Indian mustard intercropping system.

A perusal of data in Table 4 related to weed control efficiency recorded at 20,40 DAS and at harvest, revealed that the highest weed control efficiency $(61.55,70.87$ and $75.33 \%$, respectively) was noted under treatment $\mathrm{W}_{4}$, The response of different weed management practices in term of weed control efficiency at 20 DAS was remained in order of $\mathrm{W}_{4}>\mathrm{W}_{3}>$ $\mathrm{W}_{2}>\mathrm{W}_{1}>\mathrm{W}_{0}$. However, at 40 DAS and at harvest the order was $\mathrm{W}_{4}>\mathrm{W}_{2}>\mathrm{W}_{3}>\mathrm{W}_{1}>$ $\mathrm{W}_{0}$. In case of weed index, which is the indicator of losses in seed yield due to presence of weeds, the response of various weed management treatments in term of weed index was found in order of $\mathrm{W}_{4}(0.00 \%)<\mathrm{W}_{3}$ $(2.79 \%)<\mathrm{W}_{2}(8.24 \%)<\mathrm{W}_{1}(18.62 \%)<\mathrm{W}_{0}$ $(52.21 \%)$. This might be due to effective weed control achieved under efficient method of weed management in terms of lower weed population per unit area and less availability of underground (nutrient and moisture) and above ground resources (light) to weeds due to more competitive and smothering effect of crop, resulting lower biomass of weeds and higher weed control efficiency.

Ultimately, this treatment gave more yield to the crops and recorded lower weed index. Almost similar results were also reported by Sarkar et al., (2005) at West Bangal; Adhikari and Ghosh (2014) at West Bangal and Kour et al., (2014) at Jammu (J \& K). 
There was non-significant effect on nitrogen, phosphorus and potassium content in weeds due to various weed management treatments (Table 5).

Whereas, the lowest uptake of $\mathrm{N}(1.43 \mathrm{~kg}$ $\left.\mathrm{ha}^{-1}\right), \mathrm{P}\left(0.53 \mathrm{~kg} \mathrm{ha}^{-1}\right)$ and $\mathrm{K}\left(1.65 \mathrm{~kg} \mathrm{ha}^{-1}\right)$ by total weeds noticed under treatment $\mathrm{W}_{4}$ followed by treatment $\mathrm{W}_{2}$ due to their effective weed control efficiency. Weedy check $\left(\mathrm{W}_{0}\right)$ recorded the highest uptake of nitrogen, phosphorous and potassium by weeds might be due to the vigorous growth of weeds and highest weed biomass production under this treatment. The similar results were also reported by Mundra et al., (2002) in maize at Udaipur (Rajasthan) and Singh et al., (2013) in rapeseed-mustard at Varanasi (UP).

The data in Table 1 indicated that the maximum economical gain of ₹ $60458 \mathrm{ha}^{-1}$ with BCR of 3.41 was obtained under treatment $\mathrm{W}_{4}$ which was closely followed by ₹ $59587 \mathrm{ha}^{-1}$ with BCR of 3.53 under treatment $\mathrm{W}_{3}$ and by $₹ 56082 \mathrm{ha}^{-1}$ with BCR of 3.51 under treatment $\mathrm{W}_{2}$. This was attributed to greater increase in gross realization with comparatively lower cost in this treatment resulted in higher net realization and BCR. These findings are in accordance with those reported by Singh (2006) at Jodhpur (Rajasthan); Sewak et al., (2007) at Aligarh (UP); Kumar et al., (2012) at Palampur (HP) and Kour et al., (2014) at Jammu (J \& K).

\section{Interaction effect}

Combined effect of planting methods and weed management practices on mustard and weeds were found to be non-significant for all the attributing parameters except monocots and total weed population at 40 DAS. The data presented in Table 3 revealed that the treatment combination of $\mathrm{P}_{1} \mathrm{~W}_{4}(30 \mathrm{~cm} \times 10$ $\mathrm{cm}$ with normal planting along with
Pendimethalin @ $1.0 \mathrm{~kg} \mathrm{ha}^{-1}$ as PE + Quizalofop - P - ethyl @ $0.04 \mathrm{~kg} \mathrm{ha}^{-1}$ at 20 DAS + HW and IC at 40 DAS) recorded significantly lower number of monocot weeds but it was found at par with $\mathrm{P}_{1} \mathrm{~W}_{2}, \mathrm{P}_{2} \mathrm{~W}_{4}$, $\mathrm{P}_{3} \mathrm{~W}_{4}$ and $\mathrm{P}_{2} \mathrm{~W}_{2}$.

Similar trend was also observed in case of total weeds but it was remained statistically at par with treatment combinations of $\mathrm{P}_{1} \mathrm{~W}_{2}$, $\mathrm{P}_{2} \mathrm{~W}_{4}$, and $\mathrm{P}_{2} \mathrm{~W}_{2}$ only (Table 3 ). The similar results were also reported by Takim and Adereti (2012) in soybean.

From the results of one year experimentation, it can be concluded that under prevailing agro climatic conditions the higher profitable yield of rabi mustard cv. 'GDM-4' can be obtained by sowing the crop either at row spacing of 45 $\mathrm{cm} \times 10 \mathrm{~cm}$ with normal planting or $30 / 60 \mathrm{~cm}$ $\mathrm{x} 10 \mathrm{~cm}$ with paired row planting and either to apply Pendimethalin @ $1.0 \mathrm{~kg} \mathrm{ha}^{-1}$ as PE + Quizalofop - P - ethyl @ $0.04 \mathrm{~kg} \mathrm{ha}^{-1}$ at 20 $\mathrm{DAS}+\mathrm{HW}$ and IC at 40 DAS or Pendimethalin@ $1.0 \mathrm{~kg} \mathrm{ha}^{-1}$ as PE + HW and IC at 40 DAS or Pendimethalin @ $1.0 \mathrm{~kg} \mathrm{ha}^{-1}$ as PE + Quizalofop - P - ethyl @ $0.04 \mathrm{~kg} \mathrm{ha}^{-1}$ at 20 DAS under south Gujarat conditions.

\section{Acknowledgement}

The authors would like to thank to the Department of Agronomy, Navsari Agricultural University for granted approval to conduct this research trail with Institute facilities. The Head of Farm Departments are appreciated for logistic support especially with respect to fertilizers, weedicides and reliable technical support.

\section{References}

Adhikari, P. and Ghos, R. K. 2014. Integrated weed management strategies in blackgram-brinjal-mustard cropping sequence. Environment \& Ecology, 32(2 
A): 725-727.

Anonymous. 2013. Retrieved from indiatimes.com: http://times ofindia. indiatime.com/economicsurvey2013/bu dgetarticle list/11871902.cms

Anonymous. 2014. Agricultural Statistics at a glance. Directorate of Economics and Statistics, Department of Agriculture and Cooperation, Ministry of Agriculture, Govt. of India, New Delhi. p. 319.

Banga, R. S., Yadav, A. and Bisht, R. 2004. Integrated weed management in Indian mustard. Indian Journal of weed science, 36(3\&4): 224-226.

Bhowmik, B., Mitra, B. and Bhadra, K. 2014. Diversity of insect pollinators and their effect on the crop yield of Brassica juncia L., NPJ-93, from Southern West Bengal. International Journal of Recent Scientist Research, 5 (6): 1207-1213.

Chauhan, Y. S., Bhargava, M. K. and Jain, V. K. 2005. Weed management in Indian mustard (Brassica juncea). Indian Journal of Agronomy, 50 (2): 149-151.

Gomez, A. and Gomez, A. 1984. "Statistical procedure for agricultural research" 2 nd Edition, John Willey and Sons, New York.

Hussein, F. A., El-Metwally, I. M. and ElDesoki, E.R. 2008. Effect of plant spacing and weed control treatments on productivity of maize and growth of associated weeds in sandy soil. American- Eurasian Journal of Agriculture \& Environment Sciences, 4(1): 09-17.

Iraddi, V. S. 2008. Response of Mustard [Brassica juncea (L.) Czernj and Cosson] varieties to date of sowing and row spacing in northern zone of Karnataka (Doctoral dissertation, Thesis of MSc, January 2008. College of Agriculture, University of Agricultural Sciences, Dharwad).

Jackson, M. L. 1967. "Soil Chemical
Analysis". Prentice Hall of India Pvt. Ltd., New Delhi, pp. 183-192.

Kondop, S.M. and Upadhyay, U.C. 1985. A practical manual on weed control. Oxford and IBH publ. Co., New Delhi, pp.55.

Kour, R., Sharma B. C., Kumar, A., Nandan, B. and Kour, A. 2014. Effect of weed management on chickpea (Cicer arietinum) + Indian mustard (Brassica juncea) intercropping system under irrigated conditions of Jammu region. Indian Journal of Agronomy, 59(2): 242-246.

Kumar, A., Singh, B., Yashpal and Yadava, J. S. 2004. Effect of sowing time and crop geometry on tetralocular Indian mustard (Brassica juncia) under south-west Haryana. Indian Journal of Agricultural sciences, 74(11): 594-96.

Kumar, S., Kumar, A., Rana, S.S., Chander, N. and Angiras, N.N. 2012. Integrated weed management in mustard. Indian Journal of Weed Science, 44(3): 139143.

Kumar, V. and Gill, G. S. 1969. Weed index a new method for reporting weed control trails. Indian Journal of Agronomy, 16(2): 96-98.

Muhammad, A., Muhammad, A. S. and Mushtaq, S. 2012. Inter and intra row spacing effects on growth, seed yield and oil contents of white mustard (Sinapis alba L.) under rainfed conditions. Pakistan Journal of Agriculture Science, 49(1): 21-25.

Mundra, S. L., Vyas, A. K. and Maliwal, P. 2002. Effect of weed and nutrient management on nutrient uptake by maize (Zea mays) and weeds. Indian Journal of Agronomy 47(3): 378- 383.

Panse, V. G. and Sukhatme, P. V. 1967. "Statistical Methods for Agricultural Workers". ICAR, New Delhi.

Patel, S. R. 2000. Effect of planting geometry, intercropping and weed management on 
sugarcane (Saccharum hybrid) var. CoN-91132 (Guj.Cane-1). Ph.D. thesis, submitted to G.A.U., S.K. Nagar).

Pyare, R., Prasad, K., Dixit, V., Khan, N. and Sonker T. C. 2008. Effect of row spacing and sulphur on growth, yield attributes, yield and economics of mustard [Brasssica juncia (L.) Czern \& Coss]. Plant Archives, 8(2): 633-635.

Rajak, D.R., Patel, H.A., Chaudhari, K.N., Patel, N.K., Panigrahy, S. and Parihar, J. S. 2011. Spatial temporal sowing pattern of rapeseed-mustard crop in India. International Archives of the Photogrammetry, Remote Sensing and Spatial Information Sciences, Volume XXXVIII-8/W20.

Rajput, A. L. 2012. Effect of plant density, N levels and moisture conservation practices on the performance of Indian mustard (Brassica juncea) and available $\mathrm{N}$ status of soil Indian Journal of Agronomy, 57(2): 171-175.

Rathi, J. P. S., Rathi, P. K. and Singh, O. P. 2007. Studies on integrated weed management technique in chickpea with mustard intercropping system. Plant Archives, 7(2): 909-912.

Sarkar, A., Mukherjee, P. K. and Bhattacharya, P. M. 2005. Bio-efficacy of pendimethalin and fluchloralin in mustard. Indian Journal of Weed Science, 37(3\&4): 275-276.

Sewak, R., Shah, D. and Singh, A. K. 2007. Effect of weed control measures and sulphur levels on growth and yield of Indian mustard [Brassica juncea (L.)
Czern \& Coss] cv. Pusa Bold. A Journal of Multidisciplinary Advance Research, page: 73-82.

Singh, C., Singh, P. and Singh, R. 2010. Modern Techniques of Raising Field Crops. New Delhi: Oxford \& IBH publishing company Pvt. Ltd.

Singh, R. 2006. Effect of cropping sequence, seed rate and weed management on weed growth and yield of Indian mustard in western Rajasthan. Indian Journal of Weed Science, 38(1\& 2): 6972.

Singh, R. K., Singh, R. P. and Singh, M. K. 2013. Weed management in RapeseedMustard - A Review. Agriculture Reviews, 34(1): 36-49.

Singh, T., Dahiya, K. S. and Sidhu, M. S. 2006. Effect of genotype, seedling age and row spacing on performance of transplanted African mustard (Brassica carinata) under late-sown condition. Indian Journal of Agronomy, 51(3): 221-224.

Takim, F. O. and Adereti, R. O. 2012. Effect of inter row spacing and weed management practices on the performance of soybean [Glycine max (L.) Merrill] in the southern Guinea Savanna zone of Nigeria. African Journal of Agriculture Research and Dovelopment, 5(1): 76-83.

Yadav, R. P. 2004. Effect of herbicides alone and in combination with cultural methods on weed control in Indian mustard (Brassica juncea). Indian Journal of Agronomy, 49(4): 268-270.

\section{How to cite this article:}

Rameti Jangir, L.K. Arvadia and Deep Singh Rajpurohit. 2018. Effect of Different Planting Methods and Weed Management Practices on Yield and Economic of Mustard (Brassica juncea L.) and Weed Population, Weed Dry Matter Accumulation, Weed Control Efficiency, Weed Index, Nutrient Content and Uptake by Weeds. Int.J.Curr.Microbiol.App.Sci. 7(02): 2588-2599. doi: https://doi.org/10.20546/ijcmas.2018.702.315 